\title{
Indução percutânea de colágeno associada ao ácido ascórbico no tratamento de rejuvenescimento facial
}

\author{
Percutaneous induction of collagen associated with ascorbic acid in facial rejuvenation treatment \\ Inducción percutánea de colágeno asociada com ácido ascórbico em tratamientos de \\ rejuvenecimiento facial
}

Recebido: 28/06/2021 | Revisado: 05/07/2021 | Aceito: 08/07/2021 | Publicado: 18/07/2021

\author{
Amanda Charlois Pereira \\ ORCID: https://orcid.org/0000-0002-1400-8578 \\ Universidade Paulista, Brasil \\ E-mail: amiecharlois28@gmail.com \\ Francine Stefany Martins \\ ORCID: https://orcid.org/0000-0002-1634-4495 \\ Universidade Paulista, Brasil \\ martinsfrancine@outlook.com \\ Lidiane Barbosa \\ ORCID: https://orcid.org/0000-0002-1818-1968 \\ Universidade Paulista, Brasil \\ E-mail: Barbosalidiane18@hotmail.com
}

\begin{abstract}
Resumo
O envelhecimento é um processo natural, porém a busca em reverter os sinais provocados na pele é cada vez maior atualmente. Com a idade avançada, as alterações anatômicas são principalmente as mais visíveis e manifestam-se rapidamente, desta forma contribuindo para que as pessoas procurem por tratamentos estéticos que minimizem estas alterações. Objetivo: Analisar os resultados relacionados à técnica de Indução Percutânea de Colágeno e elucidar os mecanismos da aplicação, no processo de rejuvenescimento facial, utilizando Vitamina C tópica. Métodos: As pesquisas foram realizadas através de artigos científicos encontrados nos bancos de dados Google Scholar, Surgical and Cosmetic Dermatology, Pubmed e Scielo. Foram procurados artigos publicados entre os anos de 2003 a 2015. Resultados: A técnica possui baixo custo e fácil aplicação se comparada aos demais tratamentos de rejuvenescimento existentes no mercado. A espessura da agulha para o uso é mínima $(0,5 \mathrm{~mm})$, e, por isso, pode-se considerar um tratamento indolor. A maior quantidade de vitamina $\mathrm{C}$ permeada facilita a ação antioxidante, assim combatendo os radicais livres e auxilia na neocolagênese. Conclusão: Através dos estudos analisados, conclui-se que a técnica de microagulhamento aliada a Vitamina $\mathrm{C}$ se mostra eficaz pela permeação do ativo e possui efeitos positivos conta o envelhecimento cutâneo. Apesar dos estudos já apontarem resultados benéficos a estes novos tratamentos, ainda existe escassez de dados e novas pesquisas. Recomendam-se mais pesquisas que elucidem os mecanismos moleculares acerca da aplicação de vitamina $\mathrm{C}$ associada ao microagulhamento.
\end{abstract}

Palavras-chave: Envelhecimento; Microagulhamento; Vitamina C.

\begin{abstract}
Aging is a natural process, but the search for reversing the signs caused on the skin is increasing nowadays. With advancing age, anatomical changes are mainly the most sought after and manifest themselves quickly, thus contributing to people looking for aesthetic treatments that minimize these changes. Objective: To analyze the results related to the Percutaneous Collagen Induction technique and elucidate the Application components, in the process of facial rejuvenation, using topical Vitamin C. Methods: Searches were conducted through scientific articles found in the Google Scholar, Surgical and Cosmetic Dermatology, Pubmed and Scielo databases. Articles published between 2003 and 2015 were searched. Results: The technique is low cost and easy to apply when compared to other rejuvenation treatments on the market. The needle thickness for use is minimal $(0.5 \mathrm{~mm})$, and therefore it can be considered a painless treatment. The greater amount of permeated vitamin $\mathrm{C}$ facilitates the antioxidant action, thus fighting free radicals and aiding in neocollagenesis. Conclusion: Through the studies carried out, it is concluded that the microneedling technique combined with Vitamin $\mathrm{C}$ is effective for the permeation of the active and has positive effects against skin aging. Although studies have already shown beneficial results for these new treatments, there is still a lack of data and new research. Further research is recommended to elucidate the molecular mechanisms regarding the application of vitamin $\mathrm{C}$ associated with microneedling.
\end{abstract}

Keywords: Aging; Microneedlin; Vitamin C. 


\begin{abstract}
Resumen
El envejecimiento es un proceso natural, pero la búsqueda de revertir los signos provocados en la piel es cada vez mayor en la actualidad. Con el avance de la edad, los cambios anatómicos son principalmente los más visibles y se manifiestan rápidamente, contribuyendo así a que las personas busquen tratamientos estéticos que minimicen estos cambios. Objetivo: Analizar los resultados relacionados con la técnica de Inducción Percutánea de Colágeno y dilucidar los mecanismos de aplicación, en el proceso de rejuvenecimiento facial, utilizando Vitamina C tópica. Métodos: Las investigaciones se realizaron a través de artículos científicos encontrados en las bases de datos Google Scholar, Surgical And Cosmetic Dermatology, Pubmed y Scielo. Se han buscado artículos publicados entre 2003 y 2015. Resultados: La técnica es de bajo costo y fácil de aplicar en comparación con otros tratamientos de rejuvenecimiento en el mercado. El grosor de la aguja a utilizar es mínimo $(0,5 \mathrm{~mm})$ y, por tanto, puede considerarse un tratamiento indoloro. La mayor cantidad de vitamina $\mathrm{C}$ permeada facilita la acción antioxidante, combatiendo así los radicales libres y ayudando en la neocollagénesis. Conclusión: A través de los estudios analizados, se concluye que la técnica de microagujas combinada con Vitamina $\mathrm{C}$ demuestra ser efectiva al permear el activo y tiene efectos positivos contra el envejecimiento cutáneo. Aunque los estudios ya han mostrado resultados beneficiosos para estos nuevos tratamientos, todavía faltan datos y nuevas investigaciones. Se recomienda más investigación para dilucidar los mecanismos moleculares que rodean la aplicación de vitamina $\mathrm{C}$ asociada con microagujas.
\end{abstract}

Palabras clave: Envejecimiento; Microagujas; Vitamina C.

\title{
1. Introdução
}

Cada vez mais os procedimentos estéticos têm sido procurados com objetivos que vão desde a satisfação pessoal a tratamentos patológicos. Esse segmento é um dos que mais crescem no mundo, sem apresentar oscilações em épocas específicas. Dante disso, um dos tratamentos que tem se mostrado muito eficiente nos últimos tempos é a Indução Percutânea de Colágeno (IPC) ou Microagulhamento, uma técnica que consiste em lesionar as camadas mais superficiais da pele a fim de provocar uma inflamação controlada. A técnica tem como uma de suas indicações o retardo dos sinais de envelhecimento por estimular a neocolagênese e também por ter o poder de ser associada a vários ativos. Logo, ela é extremamente procurada quando se trata desta queixa. Sabe-se que o envelhecimento é um processo natural e inevitável por todos os seres humanos, e o mesmo acarreta diversas alterações morfofuncionais e também visíveis. Apesar de se tratar de um processo infalível, preocupa muitos indivíduos em relação com a saúde e a aparência física, bem como em decorrência da maior longevidade.

Durante a juventude, a face tem a forma de um trapézio invertido; o processo de envelhecimento, porém, causa mudanças nos contornos do rosto, que tende a tornar-se um quadrado. O processo de quadralização facial, como é conhecido, é explicado com base nos quatro pilares principais do envelhecimento: flacidez cutânea, ação muscular depressora, diminuição volumétrica dos compartimentos de gordura e perda da sustentação profunda em virtude do remodelamento ósseo (Coimbra, et al., 2014).

O Envelhecimento da população e a potencialização deste cenário, a pele também vem recebendo mais atenção, em termos de prevenção de neoplasias e tratamento estético dos sinais de envelhecimento cutâneo. Este processo provoca uma alteração no aspecto, por isso, o encadeamento cutâneo ocasiona diversos sinais clínicos e fisiológicos. Tais alterações estão relacionadas com a diminuição do número de células do organismo e ao funcionamento desordenado das que permanecem. Com isso, observam-se um aumento nos procedimentos estéticos faciais e corporais, sobretudo aqueles menos invasivos. (Guirro \& Guirro, 2004).

As rugas estáticas são conseqüências da fadiga das estruturas que constituem a pele, em decorrência da repetição dos movimentos e aparecem mesmo na ausência deles. As dinâmicas ou linhas de expressão surgem como conseqüência de movimentos repetitivos da mímica facial e aparecem com o movimento. Já as rugas gravitacionais são conseqüentes da flacidez da pele, culminando com a ptose das estruturas da face. (Guirro \& Guirro, 2004).

Algumas técnicas invasivas utilizadas atualmente no tratamento da pele fotoenvelhecida consistem em causar danos à derme e à epiderme a fim de promover a fibrose; no entanto, a epiderme danificada é substituída por uma epiderme que já não tem papilas dérmicas e é mais fina do que o tecido original. Esse dano causado na epiderme leva a alterações graves na derme 
(Fernandes \& Signorini, 2008).

A associação do uso da vitamina $\mathrm{C}$ a procedimentos menos invasivos, como o microagulhamento, tem sido indicada para tratamento da pele em função tanto do menor risco de complicações quanto da possibilidade de retorno precoce às atividades laborais. Isso ocorre porque o microagulhamento estimula a produção de colágeno sem provocar remoção total do epitélio, o que é observado em outras técnicas ablativas, e permite a permeação de maior quantidade de ativos na pele. (Lima, et al., 2015).

O Ácido ascórbico, conhecido como vitamina C, é uma molécula orgânica, hidrossolúvel indispensável para a hidroxilação do colágeno. Por ser uma vitamina não é sintetizada pelo organismo, tendo que ser adquirida através da dieta (Barros \& Bock, 2009).

A vitamina $\mathrm{C}$ exerce uma ação antioxidante sobre a pele, protegendo-a da ação dos radicais livres, que favorecem o envelhecimento. Além disso, o uso tópico da vitamina C estimula por reações químicas no metabolismo formação de colágeno e consequentemente a formação de um novo tecido. A vitamina $\mathrm{C}$ para humanos (ácido ascórbico) pode ser somente obtida de alimentos, como o das frutas cítricas. A luz solar e a poluição ambiental podem apagar a vitamina C presente na epiderme e, como a vitamina $\mathrm{C}$ é um potente antioxidante, parece razoável que se obtenha benefícios aumentando suas concentrações na pele. As preparações de vitamina $C$ são instáveis, por isso devem ser mantidas em recipientes herméticos e em embalagens resistentes à luz para evitar exposição aos raios UV ou ao ar. (Manela-Azulay, et al., 2003).

Os avanços atuais levaram ao surgimento de tecnologias que buscam contornar transitoriamente a função de barreira do estrato córneo, expandindo, assim, a gama de ativos que podem ser administrados transdermicamente, o que resulta no aumento da atividade terapêutica do ativo (Tiwari, et al., 2010).

Esses fatos mostram que o microagulhamento pode ser utilizado como condutor de ativos como a vitamina C, facilitando sua permeação na pele e melhorando os resultados do tratamento. Diante disto, o estudo foi feito para avaliar os mecanismos de ação e aplicação do Microagulhamento associado à vitamina $\mathrm{C}$ para retardar os sinais de envelhecimento da pele.

\section{Metodologia}

Este estudo é uma revisão bibliográfica (Estrela, 2018), sobre o uso da técnica de Microagulhamento associado à Vitamina $\mathrm{C}$ no tratamento de rejuvenescimento facial. As pesquisas foram realizadas através de artigos científicos encontrados nos bancos de dados Google Scholar, Surgical and Cosmetic Dermatology, Pubmed e Scielo. Foram procurados artigos publicados entre os anos de 2003 a 2015, utilizando-se os termos Envelhecimento, Microagulhamento e Vitamina C. Todos os estudos disponíveis envolvendo o uso do ativo foram incluídos. Os critérios de inclusão neste trabalho foram artigos na língua portuguesa e inglesa, e os critérios de exclusão foram artigos publicados antes de 2003 e que não tinham acesso livre.

\section{Resultados e Discussão}

Esta pesquisa apresenta-se como qualitativa e foi feita através de pesquisas disponíveis na literatura sobre a eficácia do ativo apresentado, associada ao tratamento de Indução Percutânea de Colágeno. A partir das bases de dados, foram selecionados artigos que abordassem a técnica utilizando vitamina $\mathrm{C}$ tópica em diversas queixas. Foram encontradas 30 citações. Após o processo de triagem dos títulos e resumos, 4 artigos foram selecionados e 26 artigos foram excluídos por não abordarem unicamente o problema central escolhido. Assim, conforme os critérios de inclusão e de exclusão, 4 artigos foram incluídos para análise qualitativa, avaliação crítica, e extração de dados na tabela abaixo. 
Tabela 1. Apresentação dos artigos que abordam a eficácia da técnica em conjunto da Vitamina C.

\begin{tabular}{|c|c|c|c|c|}
\hline Autor e ano & $\begin{array}{l}\text { Características da } \\
\text { amostra }\end{array}$ & $\begin{array}{l}\text { Características do } \\
\text { equipamento }\end{array}$ & Métodos & Resultados \\
\hline Chawla, 2014. & $\begin{array}{l}\text { Paciente com } \\
\text { cicatrizes atróficas. }\end{array}$ & $\begin{array}{l}\text { Agulhas com } 1,5 \mathrm{~mm} \\
\text { de comprimento. }\end{array}$ & $\begin{array}{l}\text { O lado esquerdo da face } \\
\text { foi tratado com } \\
\text { microagulhamento e } \\
\text { vitamina C numa } \\
\text { concentração de } 15 \% \text {. A } \\
\text { técnica foi realizada com } \\
\text { movimentos em vertical } \\
\text { e horizontal, com cerca } \\
\text { de } 4 \text { a } 5 \text { passadas por } \\
\text { região. }\end{array}$ & $\begin{array}{l}\text { Os resultados gerais foram } \\
\text { satisfatórios. A Vitamina C se } \\
\text { mostrou uma ótima opção ao } \\
\text { melhorar a firmeza da pele. }\end{array}$ \\
\hline Kalil et al. 2015. & $\begin{array}{l}\text { Pacientes com sinais } \\
\text { de envelhecimento da } \\
\text { pele da região } \\
\text { anterior do tórax. }\end{array}$ & $\begin{array}{l}\text { Agulhas com } 1,5 \mathrm{~mm} \\
\text { de comprimento. }\end{array}$ & $\begin{array}{l}\text { A técnica foi realizada } \\
\text { na região do tórax dos } \\
\text { pacientes, com cerca de } \\
10 \text { passadas por local, } \\
\text { com movimentos } \\
\text { horizontais e verticais, e } \\
\text { pressão mínima nas } \\
\text { mãos. }\end{array}$ & $\begin{array}{l}\text { Houve uma melhora de } 100 \% \text { nos } \\
\text { aspectos textura da pele, suavidade } \\
\text { e firmeza. }\end{array}$ \\
\hline Lima, 2015. & $\begin{array}{l}\text { Pacientes com rugas } \\
\text { e cicatrizes de acne } \\
\text { na face. }\end{array}$ & $\begin{array}{l}\text { Agulhas com } 2.5 \mathrm{~mm} \\
\text { de comprimento. }\end{array}$ & $\begin{array}{l}\text { A técnica foi realizada } \\
\text { com movimentos de vai } \\
\text { e vem ate a obtenção de } \\
\text { orvalho sanguíneo } \\
\text { uniforme. }\end{array}$ & $\begin{array}{l}28 \text { pacientes tratados, } 12 \\
\text { apresentavam rugas e flacidez e } 10 \\
\text { apresentavam rugas, flacidez e } \\
\text { cicatrizes acne; } 100 \% \text { dos } \\
\text { pacientes relataram satisfação com } \\
\text { os resultados. }\end{array}$ \\
\hline $\begin{array}{l}\text { Fabbrocini et al. } \\
2009 .\end{array}$ & $\begin{array}{l}\text { Pacientes com rugas } \\
\text { periorbitais. }\end{array}$ & $\begin{array}{l}\text { Agulhas com } 1,5 \mathrm{~mm} \\
\text { de comprimento. }\end{array}$ & $\begin{array}{l}\text { A técnica foi realizada } \\
\text { com anestésico tópico e } \\
\text { movimentos de vai e } \\
\text { vem por duas sessões. }\end{array}$ & $\begin{array}{l}8 \text { semanas após a primeira sessão, } \\
\text { todos os pacientes tinham a pele } \\
\text { mais suave, uma leve melhora. } 32 \\
\text { semanas após a segunda sessão, a } \\
\text { melhora se deu } 100 \% \text {. }\end{array}$ \\
\hline
\end{tabular}

Fonte: Autores (2021).

Este estudo avaliou os efeitos do microagulhamento associado à vitamina $\mathrm{C}$ no tratamento de rejuvenescimento da pele. Ativos com efeito antienvelhecimento podem ser incorporados aos cosmecêuticos, tais como vitaminas, minerais, botânicos, peptídeos e fatores de crescimento, a fim de se obterem benefícios clínicos (Bagatin, 2008).

Segundo Lima et al. (2013), a proposta do microagulhamento é preconizada pela proposta de tratamentos ablativos visando o estímulo e remodelagem do colágeno. A remoção da epiderme de forma mecânica ou química favorece a liberação de citocinas e migração de células inflamatórias que culminam na substituição do tecido danificado por um tecido cicatricial. Para confirmar o benefício da indução de colágeno do microagulhamento, estudos realizados no sul da Alemanha por Aust, et al. (2008), com indivíduos apresentando rugas, cicatrizes e estrias, foram realizadas de uma até quatro sessões de microagulhamento com aplicação tópica de vitamina A e C. A avaliação dos pacientes revelou uma melhoria de $60 \%$ a $80 \%$ na aparência da pele, exames histológicos, em 20 pacientes, demonstraram um aumento significativo de colágeno e elastina seis meses após o tratamento, e um aumento de $40 \%$ na espessura da epiderme um ano após o tratamento.

O ácido L-ascórbico pode ser transportado através do estrato córneo apenas com um pH menor que 3,5 ou por meio de tratamentos que rompem o estrato córneo, podendo melhorar sua permeação (Draelos, 2005), como o ocorre no microagulhamento. Baumann (2004) indica a adição de ácido ascórbico no tratamento da pele de pessoas que se expõem insistentemente à luz solar, ao tabagismo e aos demais comportamentos relacionados à produção de radicais livres.

Hemilä e Kaprio (2009) relatam que em um estudo realizado na Finlândia com uma população de idosos fumantes do sexo masculino, observou-se que a suplementação de vitamina E associada a uma dieta com altos níveis de vitamina C, tornouse benéfica para esse grupo populacional. Essa junção de vitaminas diminuiu os riscos de doenças relacionadas ao envelhecimento, refletindo em menores índices de mortalidade. Os benefícios na combinação dessas vitaminas ocorrem 
provavelmente, por ambas serem potentes antioxidantes, neutralizando assim processos de estresse oxidativo que são as principais causas do envelhecimento humano. Já Lima et al. (2013) verificou o uso do microagulhamento como forma de veicular ativos com Retinol e Vitamina C para fim de rejuvenescimento. Observaram também que o uso isolado dessa técnica promove melhora na textura, na coloração e no brilho de peles envelhecidas. Os autores relataram também que o microagulhamento pode ser utilizado:

A) Como veiculador de ativos para rejuvenescimento como o retinol e a vitamina $\mathrm{C}$.

B) Para estímulo isolado no rejuvenescimento da face, melhorando a coloração, textura e brilho da pele.

C) No tratamento da flacidez e atenuação de rugas, já que favorece a produção de colágeno proporcionando aumento de volume da área tratada à custa desse estímulo.

D) Na correção de cicatrizes deprimidas distensíveis, onduladas e retráteis, bem como na melhoria de estrias recentes e antigas.

Segundo Gonçalves et al. (2015), os fibroblastos para produzirem o colágeno precisam de ferro e vitamina C. A vitamina $\mathrm{C}$ está ligada na síntese de colágeno e glicosaminoglicanas, que ajudam a manter os tônus e a firmeza da derme, para que haja uma síntese de colágeno é necessário o sinergismo entre a vitamina $\mathrm{C}$ e a ingestão de proteínas com as propriedades do colágeno. Farris (2005) informou que a vitamina $\mathrm{C}$ aplicada topicamente estimula a atividade produtiva de colágeno da derme e leva à melhora clínica em pele fotoenvelhecida em relação à firmeza e suavidade. Através dos estudos de Caye, et al. (2008), o envelhecimento é inevitável, porém quando utilizado ativos como a vitamina C, esse processo pode ser amenizado.

\section{Considerações Finais}

Conclui-se que a técnica de microagulhamento aliada a Vitamina $\mathrm{C}$ se mostra eficaz pela permeação do ativo e possui efeitos positivos conta o envelhecimento cutâneo, podendo ser utilizada como tratamento de rejuvenescimento graças a sua efetividade. A técnica possui baixo custo e fácil aplicação se comparada aos demais tratamentos de rejuvenescimento existentes no mercado. A espessura da agulha para o uso é mínima $(0,5 \mathrm{~mm})$, e, por isso, pode-se considerar um tratamento indolor.

A maior quantidade de vitamina $\mathrm{C}$ permeada facilita a ação antioxidante, assim combatendo os radicais livres e auxilia na neocolagênese. Apesar dos estudos já apontarem resultados benéficos a estes novos tratamentos, ainda existe escassez de dados.

Recomendam-se mais pesquisas que elucidem os mecanismos moleculares acerca da aplicação de vitamina $\mathrm{C}$ associada ao microagulhamento. Por fim, sugerimos para futuros estudos que sejam avaliadas um número maior de pesquisas inerentes ao tema.

\section{Referências}

Aust, M. C., Reimers, K., Repenning, C. \& Stahl, F. (2008). Percutaneous collagen induction: minimally invasive skin rejuvenation without risk of hyperpigmentation-fact or fiction? Plastic and Reconstructive Surgery. 122(5), 1553-63.

Bagatin, E. (2008). Envelhecimento cutâneo e o papel dos cosmecêuticos. Boletim dermatológico UNIFESP, 5(17), 1-4.

Barros, C.M. \& Bock, P.M. (2009). Vitamina C na prevenção do envelhecimento cutâneo. Centro Universitário Metodista do Sul -IPA. https://docplayer.com.br/243702-Vitamina-c-na-prevencao-do-envelhecimento-cutaneo.html

Baumann, L. (2004). Dermatologia cosmética: princípios e práticas. Revinter, 223.

Caye, M. T., Rodrigues, S., Silva, D. \& Adriano, A. (2008). Utilização da Vitamina C nas alterações estéticas do envelhecimento cutâneo. Artigo científico (graduação em Tecnologia em Cosmetologia e Estética) - Universidade do Vale do Itajaí, http://siaibib01.univali.br/pdf/Mariluci\% 20Caye\%20e\%20Sonia\%20Rodrigues.pdf 
Research, Society and Development, v. 10, n. 8, e58310817645, 2021

(CC BY 4.0) | ISSN 2525-3409 | DOI: http://dx.doi.org/10.33448/rsd-v10i8.17645

Chawla S. (2014). Split Face Comparative Study of Microneedling with PRP Versus Microneedling with Vitamin C in Treating Atrophic Post Acne Scars. Journal of cutaneous and aesthetic surgery, 7(4), 209-212. https://doi.org/10.4103/0974-2077.150742

Coimbra, D. D., Uribe, N. C. \& Oliveira, B. S. (2014). Quadralização facial no processo do envelhecimento. Surgical \& Cosmetic Dermatology:6, 65-71. http://www.surgicalcosmetic.org.br/detalhe-artigo/318/-Facial-squaring--in-the-aging-process

Draelos, Z. D. (2005). Cosmecêuticos. Elsevier, 246.

Estrela, C. (2018). Metodologia Científica: Ciência, Ensino, Pesquisa. Editora Artes Médicas.

Fabbrocini, G., Padova, M. P., Vita, V., Fardella, N., Pastore, F. \& Tosti, A. (2009). Tratamento de rugas periorbitais por terapia de indução de colágeno. Surgical \& Cosmetic Dermatology: 1(3), 106-111. http://www.surgicalcosmetic.org.br/detalhe-artigo/24/Periorbital-wrinkles-treatment-using-collageninduction-therapy

Farris P. K. (2005). Topical vitamin C: a useful agent for treating photoaging and other dermatologic conditions. Dermatologic surgery: official publication for American Society for Dermatologic Surgery, 31(7 Pt 2), 814-818. https://doi.org/10.1111/j.1524-4725.2005.31725

Fernandes, D., \& Signorini, M. (2008). Combating photoaging with percutaneous collagen induction. Clinics in dermatology, 26(2), 192-199. https://doi.org/10.1016/j.clindermatol.2007.09.006

Gonçalves, G. R., Oliveira, M. A. S., Moreira, R. F. \& Brito, D. (2015). Benefits of collagen ingestion for human body: 8(2), 190-207. https://revistas.pucsp.br/index.php/reb/article/viewFile/18568/17395

Guirro, E. \& Guirro, R. (2004). Fisioterapia Dermato-funcional. (3a ed.), Manole.

Hemilä, H. \& Kaprio, J. (2009). Modification of the Effect of Vitamin E Supplementation on the Mortality of Male Smokers by Age and Dietary Vitamin C. Finland: Am J Epidemiology: 169(8), 946-953.

Kalil, C. L. P. V., Frainer, R. H., Dexheimer, L. S., Tonoli, R. E. \& Boff, A. L. (2015). Tratamento das cicatrizes de acne com a técnica de microagulhamento e drug delivery. Surgical \& Cosmetic Dermatology: 7(2), 144-148. http://www.dx.doi.org/10.5935/scd1984-8773.201572641

Lima, E. V. A., Lima, M. A. \& Takano, D. (2015). Microagulhamento: estudo experimental e classificação de injúria provocada. Surgical \& Cosmetic Dermatology: $5(2), \quad 110-114$. http://www.surgicalcosmetic.org.br/detalhe-artigo/261/Microagulhamento--estudo-experimental-e-classificacao-da-injuriaprovocada

Lima, E. A. (2015). Associação do microagulhamento ao peeling de fenol: uma nova proposta terapêutica em flacidez, rugas e cicatrizes da acne na face. Surgical \& Cosmetic Dermatology: 7(4), 328-331. http://www.dx.doi.org/10.5935/scd1984-8773.201573693

Manela-Azulay, M., Lacerda, C. A. M., Peres, M. A., Filgueira, A. L. \& Cuzzi, T. (2003). Vitamina C. Anais Brasileiros de Dermatologia: 78(3), $265-272$. https://doi.org/10.1590/S0365-05962003000300002

Tiwari, G., Tiwari, R., Pandey, S., Pandey, P. \& Ray, A. K. (2010). Microneedles and transdermal drug delivery: A review. Der Pharmacia Lettre, 2(2), 362369. https://www.scholarsresearchlibrary.com/abstract/microneedles-and-transdermal-drug-delivery-a-review-9526.html 